\title{
OPEN A multiband circular polarization selective metasurface for microwave applications
}

\author{
Syed Muhammad Qasim Ali Shah ${ }^{1}$, Nosherwan Shoaib ${ }^{1 凶}$, Fahad Ahmed ${ }^{1}$, Akram Alomainy ${ }^{2}$, \\ Abdul Quddious ${ }^{3}$, Symeon Nikolaou ${ }^{4}$, Muhammad Ali Imran ${ }^{5}$ \& Qammer H. Abbasi ${ }^{5 凶}$
}

In this research article, a multiband circular polarization selective (CPS) metasurface is presented. A reciprocal bi-layered metasurface is designed by introducing the chirality in the structure. The top layer of the proposed metasurface is composed of circular split-ring resonator with a cross shape structure inside it. The same structure is printed on the bottom side of the proposed metasurface by rotating it at an angle of $90^{\circ}$ to achieve chirality in the structure. The proposed metasurface is able to add CPS surface capability between 5.18 and $5.23 \mathrm{GHz}$ for $y$-polarized incident wave. For the frequency band of 5.18-5.23 GHz, the transmission goes up to $-4 \mathrm{~dB}$, while the polarization extinction ratio (PER) reaches up to $-27.4 \mathrm{~dB}$ at $5.2 \mathrm{GHz}$. Similarly, for $x$-polarized incident wave, three strategic CPS operating bands are achieved within the frequency ranges of $10.64-10.82 \mathrm{GHz}, 12.25-12.47 \mathrm{GHz}$, and 14.42-14.67 GHz. The maximum PER of 47.16 dB has been achieved for the $14.42-14.67 \mathrm{GHz}$ frequency band at $14.53 \mathrm{GHz}$. Furthermore, the response of the metasurface does not vary against oblique incidences up to $45^{\circ}$. The simple structure, angular stability, multiband and miniaturized size make this metasurface an outstanding applicant for polarization conversion and biomedical applications.

Metamaterials exhibit extraordinary properties and provide ample opportunity to control and manipulate the polarization, amplitude and phase of an electromagnetic wave. Chiral metamaterials, a special subclass of metamaterials, composed of a structure which lack mirror symmetry and its mirror image cannot be superimposed. Chirality is a significant characteristic of materials in the organic world, which exits in natural molecules like proteins, amino acids and carbohydrates ${ }^{1}$. Chiral metamaterial received significant attention since the Pendry et al. ${ }^{2}$ introduced the chirality to achieve negative refractive index ${ }^{3}$ (NRI). Although, metamaterials have many advantages, howerver, these are replaced by metasurfaces ${ }^{4}$ (i.e., $2 \mathrm{D}$ analog of metamaterial). The reason being 3D metamaterials face significant fabrication complexities, have bulky size and inherently have a very narrow bandwidth. Owing to the chirality in metasurfaces, a wide variety of remarkable electromagnetic properties can be realized such as giant gyrotropy ${ }^{5,6}$, optical activity $\left(90^{\circ}\right.$-polatization rotation $\left.{ }^{7-10}\right)$, circular dichroism ${ }^{11,12}$, polarization selectivity ${ }^{13-15}$ and asymmetric transmission ${ }^{16-20}$.

The asymmetric transmission is realized by Fedotov ${ }^{21}$ in 2006 and it has now become a focal point of research to achieve asymmetric linear-to-linear polarization (optical activity) and circular polarization selectivity. Various techniques have been reported on the asymmetric linear-to-linear polarization area through the use of bi-layered ${ }^{22,23}$ and multi-layered ${ }^{24,25}$ chiral structures. Currently, scientific community has directed attention towards realizing asymmetric linear-to-circular polarization ${ }^{26-32}$. As it is a challenging task to achieve asymmetric linear-to-circular (CPS) like the ones in ${ }^{27,28,32}$, however, multi-layered structures can be deployed where multi-band or enhanced bandwidth is required.

The multiband circular polarization selectivity was achieved using multi-layered metallic structures on substrate sheet when they only operated at normal incidence ${ }^{27,28}$. The multi-layered structures cause high transmission loss, while reflection coefficient increase due to poor wave impedance matching ${ }^{32}$. Therefore, the research trend is to achieve circular polarization selectivity by using low cost bi-layered chiral metasurfaces. The dual

\footnotetext{
${ }^{1}$ Research Institute for Microwave and Millimeter-Wave Studies (RIMMS), National University of Sciences and Technology (NUST), Islamabad 44000, Pakistan. ${ }^{2}$ School of Electronic Engineering and Computer Science, Queen Mary University of London, London E1 4NS, UK. ${ }^{3} \mathrm{KIOS}$ Research and Innovation Center of Excellence, University of Cyprus, 2109 Nicosia, Cyprus. ${ }^{4}$ Frederick Research Center (FRC) and Department of Electrical Engineering, Frederick University, 1036 Nicosia, Cyprus. ${ }^{5}$ James Watt School of Engineering, University of Glasgow, Glasgow G12 800, UK. email: nosherwan.shoaib@seecs.edu.pk; nosherwan.shoaib@rimms.nust.edu.pk; qammer.abbasi@glasgow.ac.uk
} 
band circular polarization selective metasurfaces using bi-layered structure has been demonstrated ${ }^{29-32}$. Zhang et al. achieved circular polarization selectivity at two bands by using bi-layered inverted G-shaped structure s1 $^{31}$.

Khan et al. proposed a highly efficient chiral metasurface ${ }^{32}$ in which CPS operation has been achieved only at the resonance frequency of $14.79 \mathrm{GHz}$ with transmission magnitude of $-4.5 \mathrm{~dB}$, however, the design worked only for normal incidence. From the aforementioned literature, it can be realized that the designs are either low efficient, operate only at the resonance frequencies with narrow bandwidth, or work at normal incidence. In this context, achieving high efficient multiband circular polarization selectivity along with the angular stability can be attractive due to its capability to integrate with various practical applications in polarization manipulation and biomedical devices ${ }^{33,34}$.

In this paper, a novel and efficient bi-layered metasurface is presented with multiband CPS characteristics. The circular polarization selectivity is achieved within the frequency range of $5.18-5.23 \mathrm{GHz}$ for y-polarization. While for $\mathrm{x}$-polarization, the CPS operation is achieved in three frequency bands, i.e., $10.64-10.82 \mathrm{GHz}$, 12.25-12.47 GHz and 14.42-14.67 GHz. For C-band (5.18-5.23 GHz), transmission goes upto $-4 \mathrm{~dB}$ which is the highest magnitude that has been achieved till to date to the best of authors' knowledge. Moreover, the metasurface operates very well for both $\mathrm{x}$-polarizaton (TM) and y-polarization (TE) against oblique incidence up to $45^{\circ}$, offering assistance in polarization conversion applications.

\section{Theoretical analysis}

Let us consider that a plane wave is travelling along $-\mathrm{z}$ direction whose incident electric field $\left(E_{i}\right)$ and transmitted electric field $\left(E_{t}\right)$ can be stated $\mathrm{as}^{35}$ :

$$
\begin{aligned}
& E_{i}(x, y, z, t)=\left[\begin{array}{c}
E_{x i} \\
E_{y i}
\end{array}\right] e^{-i(k z-w t)} \\
& E_{t}(x, y, z, t)=\left[\begin{array}{c}
E_{x t} \\
E_{y t}
\end{array}\right] e^{-i(k z-w t)}
\end{aligned}
$$

where $w$ and $k$ represent the frequency and wave number, respectively, while the complex amplitudes $\left(E_{x}\right.$ and $\left.E_{y}\right)$ represent the $\mathrm{x}$-and $\mathrm{y}$-components of electric field. The transmission matrix (T-matrix) for linear polarization can be defined $a^{36}$ :

$$
\left[\begin{array}{c}
E_{x t} \\
E_{y t}
\end{array}\right]=\left[\begin{array}{ll}
T_{x x} & T_{x y} \\
T_{y x} & T_{y y}
\end{array}\right]\left[\begin{array}{c}
E_{x i} \\
E_{y i}
\end{array}\right]=T \cdot C_{\text {Linear }}\left[\begin{array}{c}
E_{x i} \\
E_{y i}
\end{array}\right]
$$

Here $T_{x x}$ and $T_{x y}$ represent the co- and cross-component of transmission for x-polarized wave, respectively. While $T_{y x}$ and $T_{y y}$ indicates the cross- and co-component of transmission for y-polarized wave, respectively. The transmission component (T.C) can be written as T.C $=E_{t} / E_{i}$. Moreover, the transmission matrix (T-matrix) for circular polarization can be expressed $\mathrm{as}^{36}$ :

$$
\left[\begin{array}{c}
E_{+t} \\
E_{-t}
\end{array}\right]=\left[\begin{array}{ll}
T_{+x} & T_{+y} \\
T_{-x} & T_{-y}
\end{array}\right]\left[\begin{array}{c}
E_{x i} \\
E_{y i}
\end{array}\right]=T \cdot C_{c i r}\left[\begin{array}{c}
E_{x i} \\
E_{y i}
\end{array}\right]
$$

Here + and - represent the right-hand circular polarization (RHCP) and left-hand circular polarization (LHCP), respectively. The T.C $C_{\text {cir }}$ matrix can be transformed to the Cartesian basis such as ${ }^{27,32}$ :

$$
T_{c i r}=\left[\begin{array}{ll}
T_{+x} & T_{+y} \\
T_{-x} & T_{-y}
\end{array}\right]=\frac{1}{\sqrt{2}}\left[\begin{array}{ll}
T_{y x}-i T_{x x} & T_{y y}+i T_{x y} \\
T_{x x}-i T_{y x} & T_{y y}-i T_{x y}
\end{array}\right]
$$

\section{Simulated results}

Design of CPS metasurface. The schematic view of the proposed circular polarization selective metasurface along with the top and bottom layers of the unit cell are presented in Fig. 1. The designed metasurface is printed on both sides of the Rogers RT5870 substrate $\left(\varepsilon_{r}=2.33\right.$, loss tangent $\left.=0.0012\right)$ having thickness of $1.57 \mathrm{~mm}$. The $17 \mu \mathrm{m}$ copper cladding (with a conductivity of $5.80 \times 10^{7} \mathrm{~S} / \mathrm{m}$ ) is employed to design the metallic split-ring resonator (SRR) and cross-element resonator. The design on the back side of the substrate is rotated by an angle of $90^{\circ}$ compared to the front side of the substrate to achieve chirality in the structure. The unit cell is repeated itself across the $\mathrm{x}-\mathrm{y}$ plane with the same period of $10 \mathrm{~mm}$ to generate an array. To optimize the proposed design, its dimensions are varied with the intention to obtain desired results. The optimized parameters of the unit cell are as follows: $\mathrm{p} 1=10, \mathrm{p} 2=10, \mathrm{a}=4.5, \mathrm{~b}=3.55, \mathrm{~d}=0.75, \mathrm{c}=5$ and $\mathrm{g}=0.75$ (all units in mm).

To simulate and analyze the proposed metasurface, a full electromagnetic numerical solver, CST Studio Suite is utilized. To simulate the metasurface, periodic and open boundary conditions along $\mathrm{x}$ - and $\mathrm{y}$-directions along with Floquet ports along z-direction are used. The simulated transmission components (i.e., $T_{y y}, T_{x y}, T_{y x}, T_{x x}$ ) for both transverse-electric (TE) and transverse-magnetic (TM) incident waves propagating along the backward $(-z)$ and forward $(+z)$ directions are presented in Fig. 2a,b, respectively. For y-polarization, a CPS operating band is achieved within the frequency band of $5.18-5.23 \mathrm{GHz}$ while three frequency bands of the circular polarization selectivity ranging from 10.64 to $10.82 \mathrm{GHz}, 12.25$ to $12.47 \mathrm{GHz}$ and 14.42 to $14.67 \mathrm{GHz}$ are realized for $\mathrm{x}$-polarization in the backward direction as displayed in Fig. 2a. On the other hand, it can be manifested from Fig. $2 b$ that no CPS operating band is achieved over these frequency bands: $5.18-5.23 \mathrm{GHz}, 10.64-10.82 \mathrm{GHz}$, $12.25-12.47 \mathrm{GHz}$ and $14.42-14.67 \mathrm{GHz}$ when either $\mathrm{x}$-polarization (TM) or y-polarization (TE) is incident from 


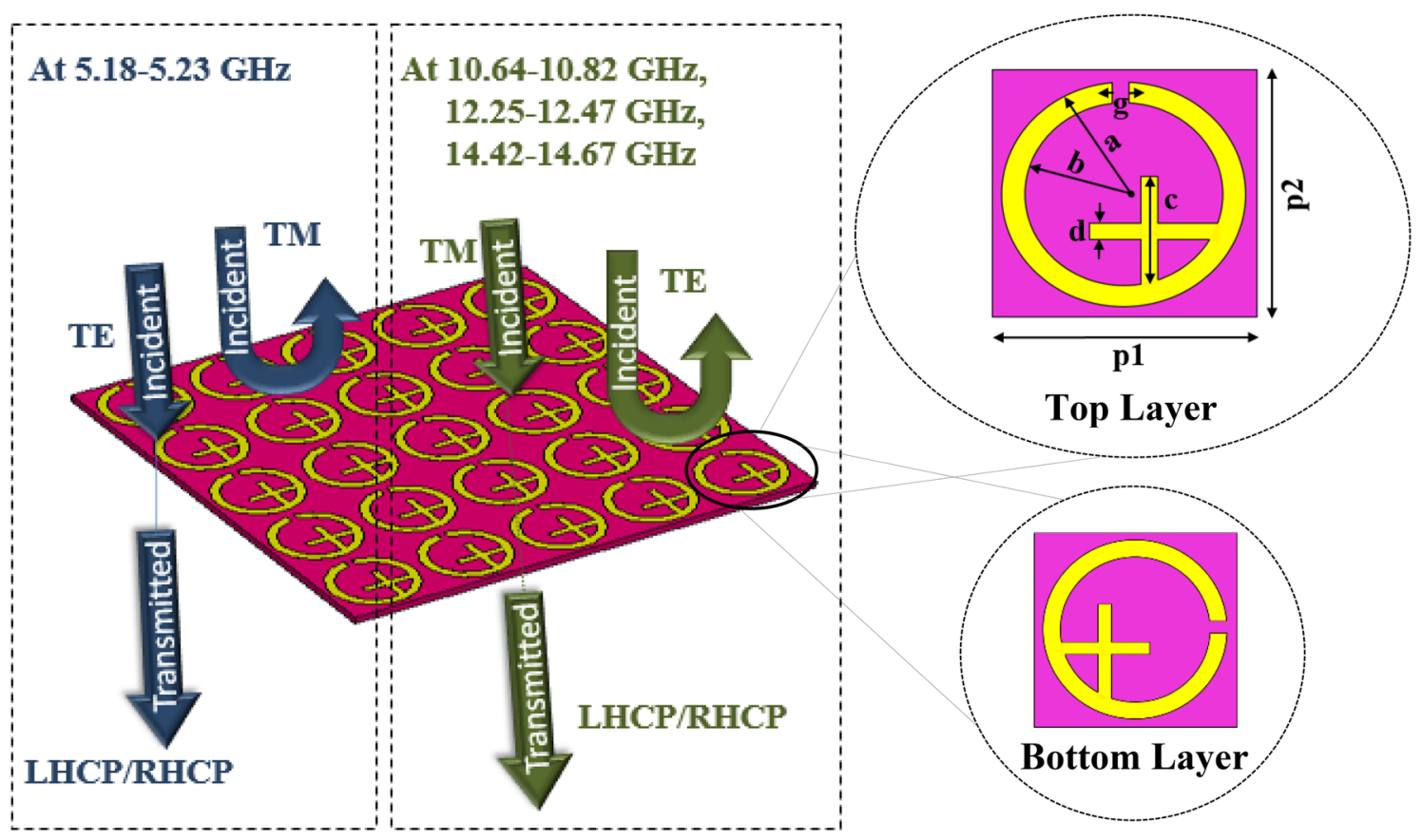

Figure 1. Schematic diagram of the circular polarization selective metasurface along with geometric configuration of the top and bottom metallic layers of the unit cell.

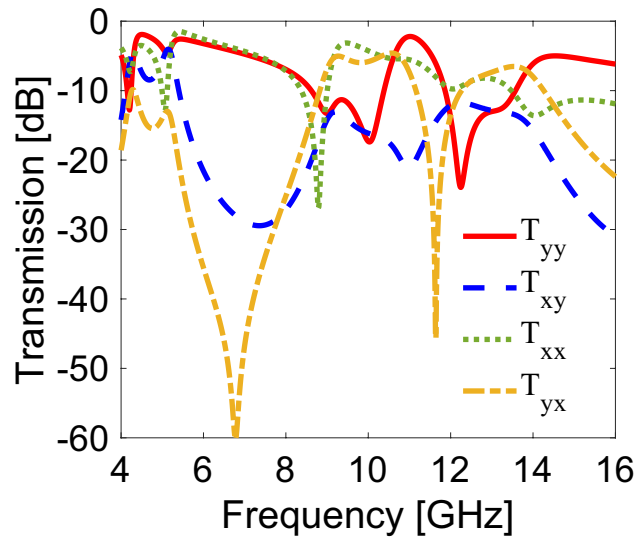

(a)

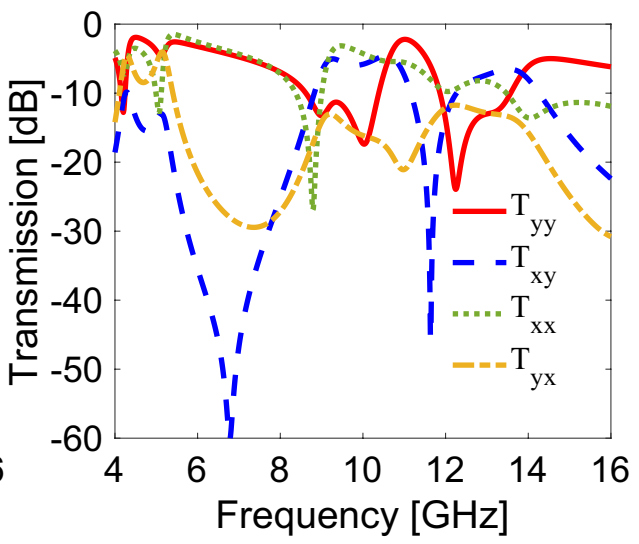

(b)

Figure 2. Magnitude of co- and cross-transmission components in (a) backward direction, (b) forward direction.

the forward direction. For instance, from Fig. $2 a$, it can be realized that the co- $\left(T_{y y}\right)$ and cross- $\left(T_{x y}\right)$ components of the TE wave are approximately equal with transmission magnitude of about $0.63(-4.0 \mathrm{~dB})$ at $5.2 \mathrm{GHz}$ while in Fig. 2b, transmission components $\left(T_{y y}\right.$ and $T_{x y}$ ) are not equal at $5.2 \mathrm{GHz}$, leading to no CPS operation in the forward direction. Similarly, in the case of TM wave, transmission components $\left(T_{x x}\right.$ and $\left.T_{y x}\right)$ are not equal over three claimed bands in the forward direction.

The CPS operating bands are achieved when magnitude ratio and phase difference between co- and crosscomponents lie within $(0.85-1.15)$ and $\left(85^{\circ}-95^{\circ}\right)$, respectively. In Fig. 3a, the magnitude ratio ${ }^{37}\left(T_{x y} / T_{y y}\right)$ remains within $(1 \pm 0.15)$ while the phase difference ${ }^{37}\left(\varphi_{\text {diff }}=\varphi_{x y}-\varphi_{y y}\right)$ between 5.18 and $5.23 \mathrm{GHz}$ remains nearly $90^{\circ}$ as indicated in Fig. 3b. Therefore, pure circular polarization selectivity is achieved within $5.18-5.23 \mathrm{GHz}$ when y-polarization is incident. Similarly, when $x$-polarization is incident on the metasurface, the magnitude ratio $\left(T_{y x} /\right.$ $\left.T_{x x}\right)$ remains nearly equal to 1 and their phase difference $\left(\varphi_{d i f f}=\varphi_{y x}-\varphi_{x x}\right)$ remains around $\pm 90^{\circ}$ or odd multiples of $\pm 90^{\circ}$ over the frequency ranges of $10.64-10.82 \mathrm{GHz}, 12.25-12.47 \mathrm{GHz}$ and $14.42-14.67 \mathrm{GHz}$. From this, it can be stated that the pure circular polarization selectivity is achieved at all aforementioned frequency bands when TM wave is incident. 


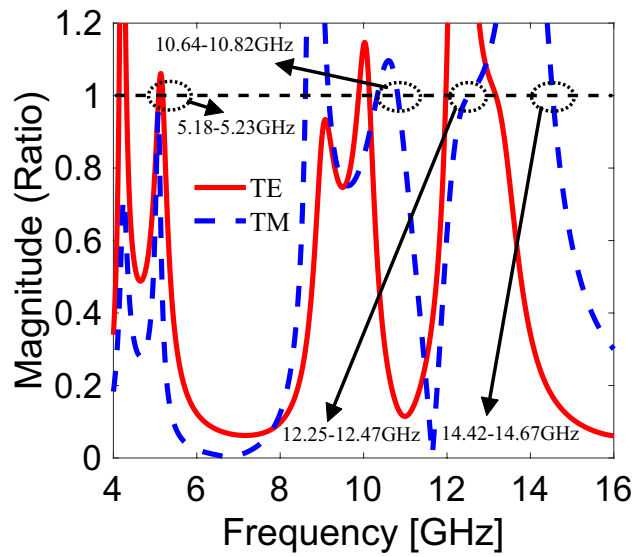

(a)

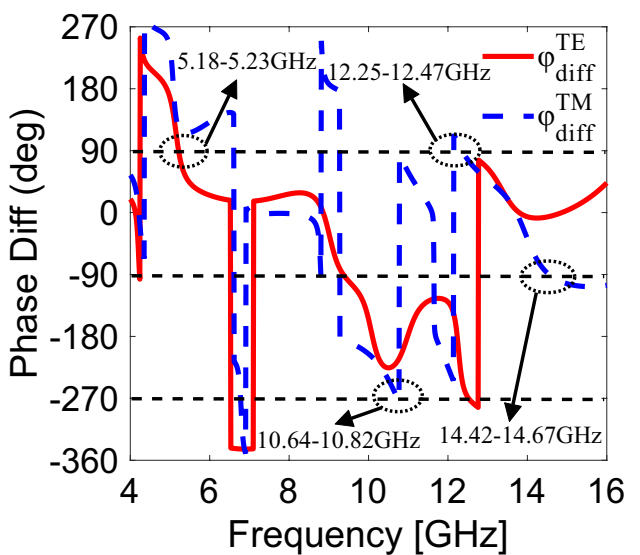

(b)

Figure 3. For both y-polarization and x-polarization, (a) magnitude ratio, (b) phase difference between co- and cross-components.

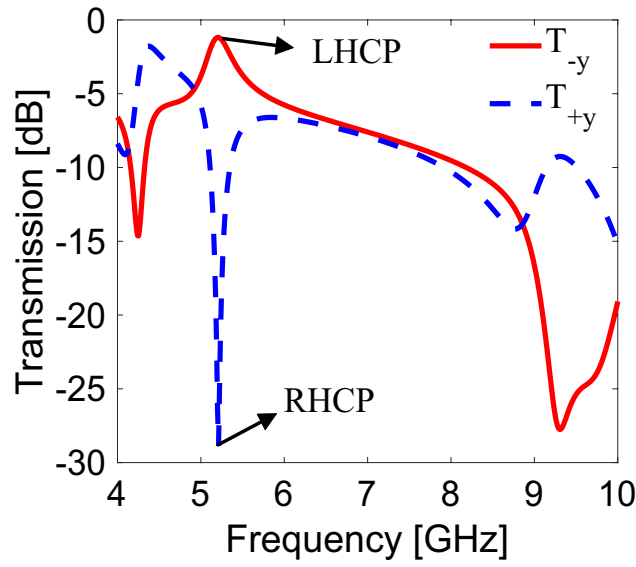

(a)

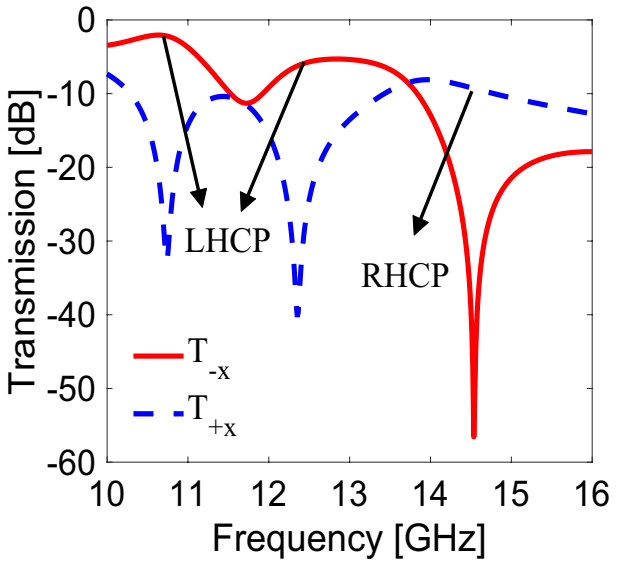

(b)

Figure 4. Circular polarization transmission components for (a) y-polarization, (b) x-polarization.

Circular polarization transmission. The transmission components for circular polarization (T. $\left.C_{c i r}\right)$ can be obtained by using T. $C_{\text {Linear }}$ components. T. $C_{\text {cir }}$ can be calculated for both $\mathrm{x}$ - and y-polarizations by using Eq. (5). From Fig. 4a, for y-polarized wave, the circular polarization transmission for LHCP has a maximum value of $-1.18 \mathrm{~dB}$ while for RHCP, it has a minimum value of $-28.7 \mathrm{~dB}$ at $5.21 \mathrm{GHz}$. There is a remarkable difference between the values of LHCP and RHCP at $5.21 \mathrm{GHz}$ such that LHCP is permitted while RHCP is restricted to pass through the metasurface at $5.21 \mathrm{GHz}$. This indicates that the incident $\mathrm{y}$-polarization is converted to the pure LHCP at $5.21 \mathrm{GHz}$. Similarly, for x-polarized wave, the circular polarization transmission for LHCP reaches up to values of $-2.17 \mathrm{~dB}$ at $10.74 \mathrm{GHz},-6.23 \mathrm{~dB}$ at $12.35 \mathrm{GHz}$ and $-56.5 \mathrm{~dB}$ at $14.53 \mathrm{GHz}$, respectively, as shown in Fig. $4 \mathrm{~b}$. On the other hand, circular polarization transmission for RHCP has minimum values of $-32.6 \mathrm{~dB}$ at $10.74 \mathrm{GHz},-40.19 \mathrm{~dB}$ at $12.35 \mathrm{GHz}$ and has a value of $-9.29 \mathrm{~dB}$ at $14.53 \mathrm{GHz}$. It shows that a pure LHCP is obtained at $10.74 \mathrm{GHz}$ and $12.35 \mathrm{GHz}$ while a pure RHCP is achieved at $14.53 \mathrm{GHz}$ when $\mathrm{x}$-polarization is incident.

Polarization extinction ratio. Polarization extinction ratio (PER) is another criterion to demonstrate the functionality of polarization conversion. It is a parameter that verifies the efficiency of circular polarization selectivity. For y-polarization, PER can be calculated by using Eq. $(6)^{37}$.

$$
\text { PER }=20 * \log _{10} \frac{\left|T_{+y}\right|}{\left|T_{-y}\right|}
$$

To find PER for x-polarization, $T_{+y}$ and $T_{-y}$ need to be replace with $T_{+x}$ and $T_{-x}$, respectively. For pure CPS operation, the graph of PER should be greater than $+20 \mathrm{~dB}$ or less than $-20 \mathrm{~dB}$. In Fig. $5 \mathrm{a}$ for y-polarization, PER 


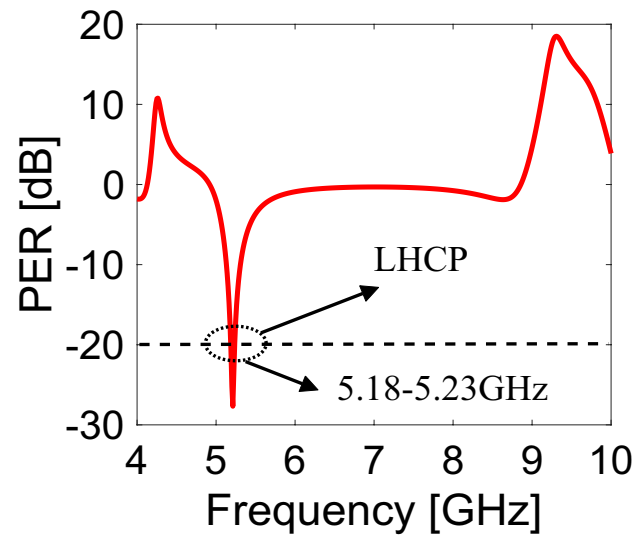

(a)

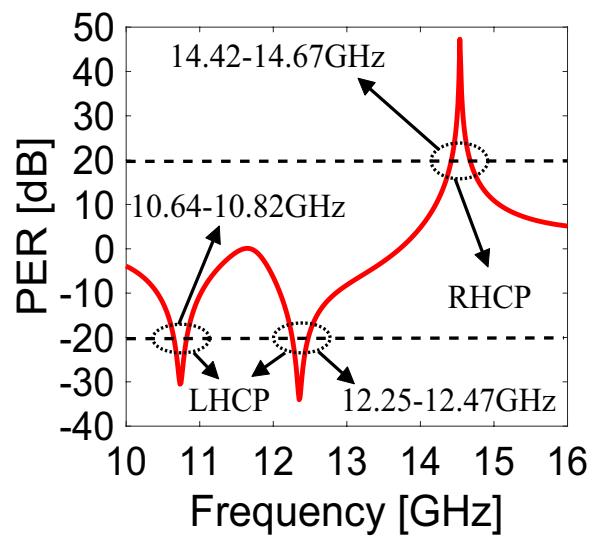

(b)

Figure 5. Polarization extinction ratio for (a) y-polarization, (b) x-polarization.

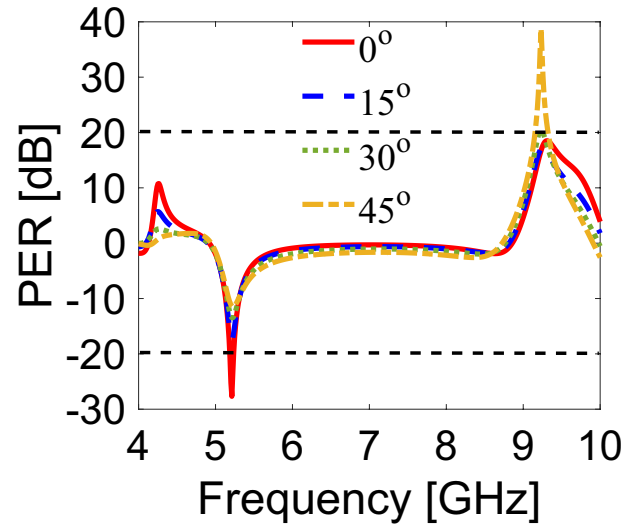

(a)

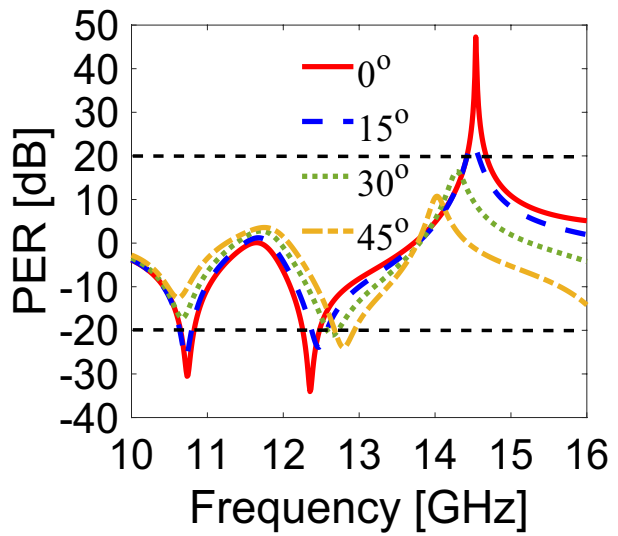

(b)

Figure 6. Polarization extinction ratio at different angles for (a) y-polarization, (b) x-polarization.

remains below $-20 \mathrm{~dB}$ in the frequency band of $5.18-5.23 \mathrm{GHz}$ with the amplitude of PER goes up to $-27.4 \mathrm{~dB}$ at $5.21 \mathrm{GHz}$. This explains that the TE wave is converted to LHCP in the frequency band of 5.18-5.23 GHz. Similarly, in Fig. 5b for x-polarization, PER remains below $-20 \mathrm{~dB}$ in the frequency bands of $10.64-10.82 \mathrm{GHz}$ and $12.25-12.47 \mathrm{GHz}$ while it remains above $+20 \mathrm{~dB}$ in the frequency band of $14.42-14.67 \mathrm{GHz}$. The amplitudes of PER reaches up to $-30.4 \mathrm{~dB}$ and $-33.9 \mathrm{~dB}$ at $10.74 \mathrm{GHz}, 12.35 \mathrm{GHz}$, respectively, while at $14.53 \mathrm{GHz}$, the amplitude of PER goes up to $47.16 \mathrm{~dB}$ which is the highest amplitude of PER that has been achieved till to date to the best of authors' knowledge. This indicates that the TM wave is converted to LHCP in the frequency bands of $10.64-10.82 \mathrm{GHz}$ and $12.25-12.47 \mathrm{GHz}$ and RHCP in the frequency band of $14.42-14.67 \mathrm{GHz}$.

Angular stability. The behavior of the CPS metasurface is analyzed at different incidence angles because of the stability requirement for many applications. It can be seen from Fig. 6 a that the proposed structure is angularly stable up to an oblique incidence of $45^{\circ}$ for y-polarization. Similarly, for x-polarization, the metasurface remains stable up to $45^{\circ}$ for the first band $(10.64-10.82 \mathrm{GHz})$ while for the second $(12.25-12.47 \mathrm{GHz})$ and third band $(14.42-14.67 \mathrm{GHz})$, it remains stable up to $15^{\circ}$ as presented in Fig. $6 \mathrm{~b}$. The response of the designed metasurface is changed at $30^{\circ}$ and $45^{\circ}$ for the two bands $(12.25-12.47 \mathrm{GHz}, 14.42-14.67 \mathrm{GHz})$ because of the larger electrical size of the unit cell. Moreover, it is interesting to see that for y-polarization, there is an additional band at resonant frequency of $9.23 \mathrm{GHz}$ which is also performing CPS operation as shown in Fig. $6 \mathrm{a}$.

Surface currents analysis. The physical behaviour behind the circular polarization selectivity can be explained by analysing the surface currents. The circular polarization conversion is the outcome of interlayer (transverse) magnetic dipole to magnetic dipole coupling at the resonances. The electromagnetic waves with in the metallic layers can be shown by eigenmodes of the resonators ${ }^{28,32}$. Figure 7 describes the induced surface currents at multiple frequencies, in which (a), (b) correspond for incident TE wave, while (c)-(h) correspond for incident TM wave. From Fig. 7a,b, it can be manifested that the surface currents on the top and bottom lay- 


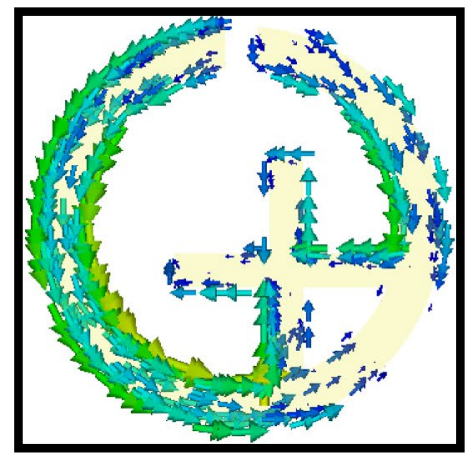

(a) Top layer at $\mathrm{f}=5.21 \mathrm{GHz}$

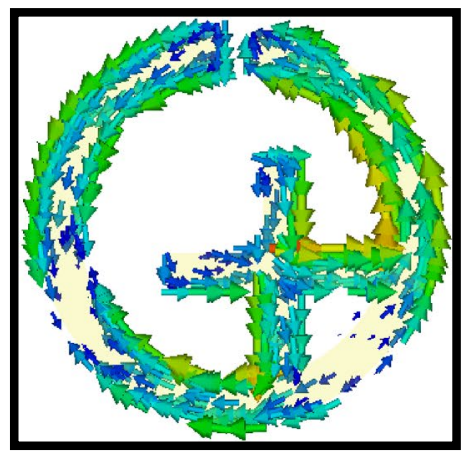

(c) Top layer at $\mathrm{f}=10.74 \mathrm{GHz}$

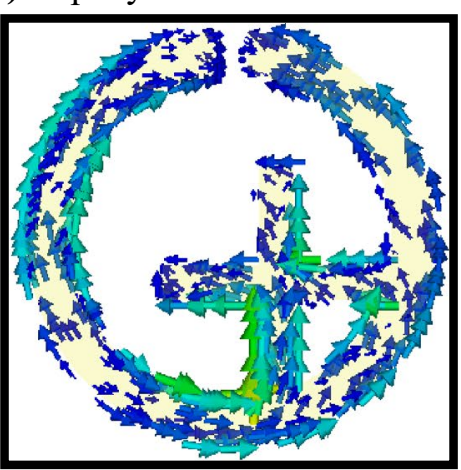

(e) Top layer at $\mathrm{f}=12.35 \mathrm{GHz}$

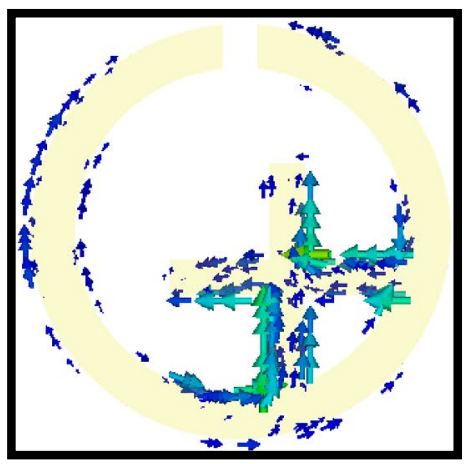

(g) Top layer at $\mathrm{f}=14.53 \mathrm{GHz}$

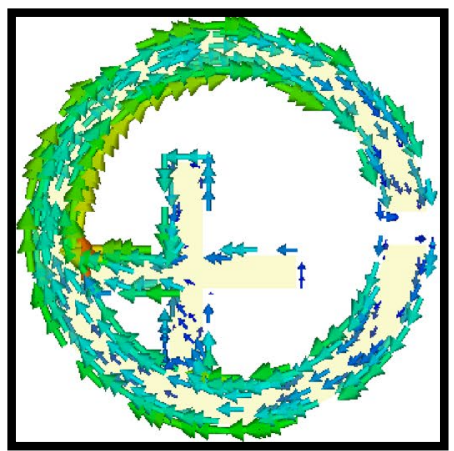

(b) Bottom layer at $\mathrm{f}=5.21 \mathrm{GHz}$

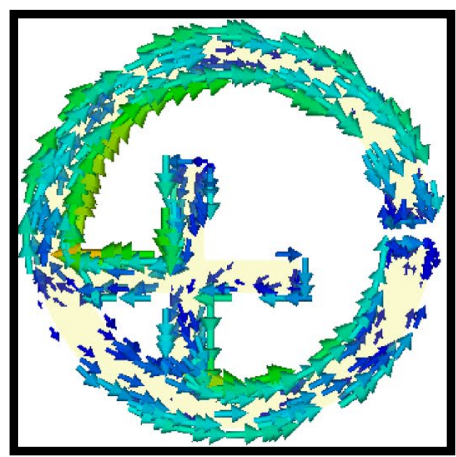

(d) Bottom layer at f $=10.74 \mathrm{GHz}$

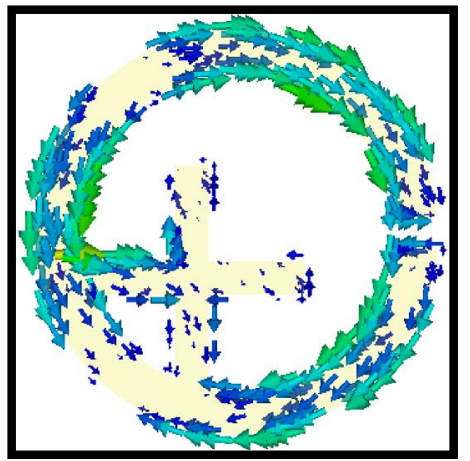

(f) Bottom layer at $\mathrm{f}=12.35 \mathrm{GHz}$

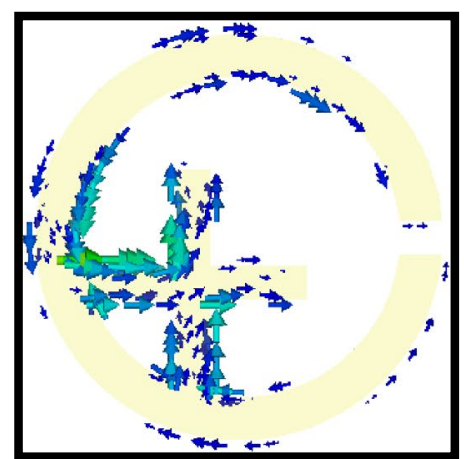

(h) Bottom layer at $\mathrm{f}=14.53 \mathrm{GHz}$

Figure 7. Surface current distributions on the top and bottom layers of the proposed metasurface at different frequencies. 


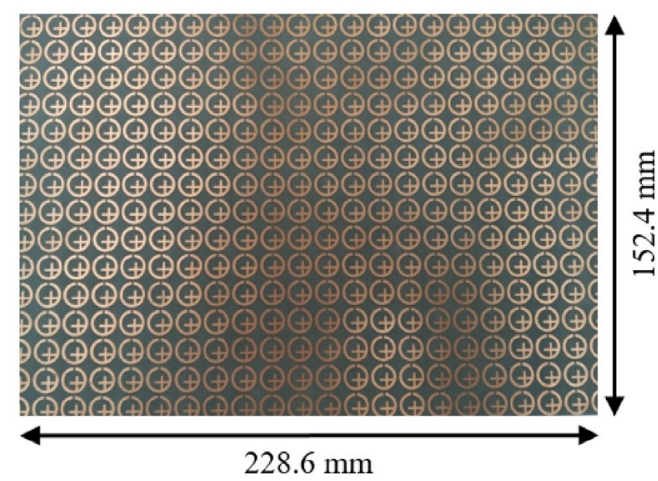

(a)

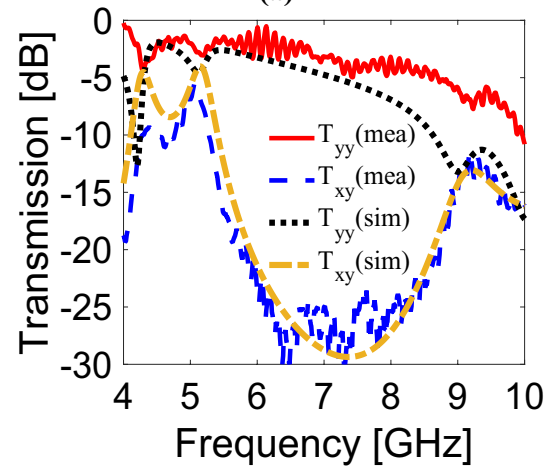

(c)

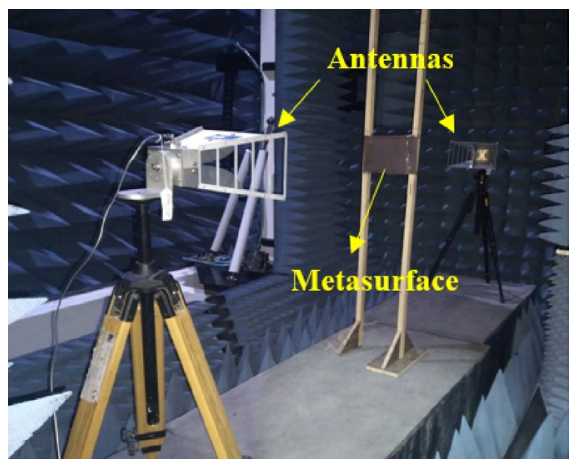

(b)

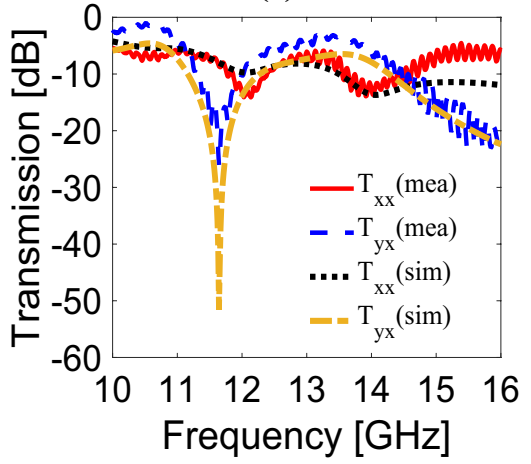

(d)

Figure 8. (a) Photograph of the fabricated metasurface, (b) experimental measurements setup, (c) comparison between simulated and measured results for TE wave, (d) comparison between simulated and measured results for TM wave.

ers of the SRR design at $5.21 \mathrm{GHz}$ are in the opposite (antiparallel) direction which show antiparallel magnetic dipole coupling ${ }^{32}$ along the external electric field. Moreover, the surface current is in parallel direction on one metallic strip while it is in antiparallel direction on the other metallic strip of the bottom layer with respect to the top layer. Thus creating the cross coupling between the electric and magnetic fields in the bi-layered chiral metasurface $^{28}$. The current directions on the top and bottom layers of the structure evaluate the type of the transmitted wave (i.e., RHCP or LHCP). Therefore, at $5.21 \mathrm{GHz}$, the antiparallel currents on the two layers depicts that the transmitted wave is LHCP. Furthermore, it is apparent from Fig. 7c,d that the antiparallel and parallel surface currents exist on the SRR and metallic strips of the bottom layer with respect to the top layer respectively, leading to an LHCP wave at $10.74 \mathrm{GHz}$ for incident TM wave. Similarly, Fig. 7e,f show that the directions of surface currents on the structure represent that LHCP wave is transmitted at $12.35 \mathrm{GHz}$ for TM wave. In addition, from Fig. 7g,h, for incident TM wave at $14.53 \mathrm{GHz}$, the surface currents on SRR structure and both metallic strips are in same (parallel) direction which show a parallel magnetic dipole coupling ${ }^{32}$ along the external electric field. Therefore, it can be stated that the transmitted wave is RHCP at $14.53 \mathrm{GHz}$.

\section{Experimental results and discussion}

In order to validate the simulated results, the proposed CPS metasurface was fabricated on Rogers 5870 substrate. The fabricated sample (having cross-section of $228.6 \times 152.4 \mathrm{~mm}^{2}$ ) consists of $22 \times 15$ unit cells, as shown in Fig. $8 \mathrm{a}$. The measurements were carried out in an anechoic chamber while the Rogers sheet was placed between two horn antennas as presented in Fig. 8b. These antennas were attached to the Anritsu-MS46122B (vector network analyzer) via coaxial cables. In order to achieve co-polarized transmission $\left(T_{y y}\right.$ or $\left.T_{x x}\right)$, both transmitting and receiving antennas were placed co-polarized, either vertical (for $T_{y y}$ ) or horizontal (for $T_{x x}$ ). To measure crosspolarized transmission, the two antennas were placed orthogonal to each other, i.e., the transmitting antenna was vertically while the receiving antenna was placed horizontally. For the measurements of the transmission components, the free space method was used through the following expression ${ }^{38}$ :

$$
S_{21}^{\text {samplecallibration }}=\frac{S_{21}^{\text {sample }}-S_{21}^{\text {metal }}}{S_{21}^{\text {air }}-S_{21}^{\text {metal }}} e^{-j(w / c) d}
$$

where $w, \mathrm{c}$ and d represent the angular frequency, speed of light and thickness of the sample, respectively. Figure $8 \mathrm{c}, \mathrm{d}$ show that the simulated and experimental results are in good agreement. The measured phase difference between co- and cross-components remains within $90^{\circ} \pm 10^{\circ}$ in all operating bands for CPS. Small discrepancies 


\begin{tabular}{|l|l|l|l|l|l|}
\hline References & Maximum PER (dB) & Angular stability & No. of bands & No. of layers & Thickness $(\mathbf{m m})$ \\
\hline 26 & -30.1 & No & 2 & 2 & 0.008 \\
\hline 28 & 30.1 & No & 4 & 3 & 1.1 \\
\hline 27 & 21.45 & No & 3 & 3 & 1.2 \\
\hline 29 & $<10$ & No & 1 & 2 & 1.5 \\
\hline 30 & -21.1 & No & 2 & 2 & 1.5 \\
\hline 31 & 20.74 & No & 2 & 2 & 1.5 \\
\hline 32 & 37.3 & No & 1 & 2 & 1.524 \\
\hline This work & 47.16 & Up to $45^{\circ}$ & 4 & 2 & 1.57 \\
\hline
\end{tabular}

Table 1. Comparison of different CPS metasurfaces.

between simulated and measured results may be attributed to cable losses, calibration discontinuities, slight misplacement of antennas, and finite size of fabricated sample ${ }^{39}$.

Table 1 compares the performance of the proposed circular polarization selective metasurface with some previously reported bi-layered or tri-layered structures in terms of maximum polarization extinction ratio, angular stability, number of bands, number of layers and thickness. Improvement can be clearly seen in terms of PER, angular stability and number of bands but the proposed CPS metasurface has a larger thickness compared to other reported work listed in Table 1.

\section{Conclusion}

This paper presents a chiral metasurface which performs multiband asymmetric linear-to-circular polarization. The CPS metasurface design has an array of split ring resonators with a cross-shaped structure inside it. It is shown that the bi-layered metasurface has the ability to achieve circular polarization selectivity at frequency band between 5.18 and $5.23 \mathrm{GHz}$ for a normally incident TE polarized wave and also achieve CPS operation at different range of frequencies of $10.64-10.82 \mathrm{GHz}, 12.25-12.47 \mathrm{GHz}$ and $14.42-14.67 \mathrm{GHz}$ for a normally incident TM polarized wave. For $5.18-5.23 \mathrm{GHz}$ frequency band, the transmission goes up to $-4 \mathrm{~dB}$ at $5.2 \mathrm{GHz}$ while for the frequency band of $14.42-14.67 \mathrm{GHz}$, PER goes up to $47.16 \mathrm{~dB}$ at $14.53 \mathrm{GHz}$, which are the maximum values that has been achieved till to date to the best of authors' knowledge. Owing to the miniaturization in unit cell size, polarization selectivity and angular stability up to $45^{\circ}$, the designed metasurface can be suitable for polarization conversion and biomedical applications.

\section{Data availability}

The datasets generated during and/or analyzed during the current study are available from the corresponding author on reasonable request.

Received: 21 October 2020; Accepted: 31 December 2020

Published online: 19 January 2021

\section{References}

1. Li, Z., Liu, W., Cheng, H., Chen, S. \& Tian, J. Spin-selective transmission and devisable chirality in two-layer metasurfaces. Sci. Rep. 7, 8204 (2017).

2. Pendry, J. B. A chiral route to negative refraction. Science (80-) 306, 1353-1355 (2004).

3. Zhou, J. et al. Negative refractive index due to chirality. Phys. Rev. B 79, 121104 (2009).

4. Shah, S. M. Q. A., Ahmed, F. \& Shoaib, N. A Highly efficient multifunctional metasurface for C-and X-band applications. In 2020 14th European Conference on Antennas and Propagation (EuCAP) 1-4 (2020). https://doi.org/10.23919/EuCAP48036.2020.91357 16.

5. Rogacheva, A. V., Fedotov, V. A., Schwanecke, A. S. \& Zheludev, N. I. Giant gyrotropy due to electromagnetic-field coupling in a bilayered chiral structure. Phys. Rev. Lett. 97, 177401 (2006).

6. Taravati, S., Khan, B. A., Gupta, S., Achouri, K. \& Caloz, C. Nonreciprocal nongyrotropic magnetless metasurface. IEEE Trans. Antennas Propag. 65, 3589-3597 (2017).

7. Plum, E., Fedotov, V. A. \& Zheludev, N. I. Optical activity in extrinsically chiral metamaterial. Appl. Phys. Lett. 93, 191911 (2008).

8. Decker, M., Zhao, R., Soukoulis, C. M., Linden, S. \& Wegener, M. Twisted split-ring-resonator photonic metamaterial with huge optical activity. Opt. Lett. 35, 1593-1595 (2010).

9. Song, K., Zhao, X., Liu, Y., Fu, Q. \& Luo, C. A frequency-tunable $90^{\circ}$-polarization rotation device using composite chiral metamaterials. Appl. Phys. Lett. 103, 101908 (2013).

10. Song, K. et al. Planar composite chiral metamaterial with broadband dispersionless polarization rotation and high transmission. J. Appl. Phys. 120, 245102 (2016).

11. Cheng, Y., Nie, Y., Wu, L. \& Gong, R. Z. Giant circular dichroism and negative refractive index of chiral metamaterial based on split-ring resonators. Prog. Electromagn. Res. 138, 421-432 (2013).

12. Kwon, D.-H., Werner, P. L. \& Werner, D. H. Optical planar chiral metamaterial designs for strong circular dichroism and polarization rotation. Opt. Express 16, 11802-11807 (2008).

13. Lončar, J., Grbic, A. \& Hrabar, S. Ultrathin active polarization-selective metasurface at X-band frequencies. Phys. Rev. B 100, 75131 (2019). 
14. Li, Y. et al. Wideband selective polarization conversion mediated by three-dimensional metamaterials. J. Appl. Phys. 115, 234506 (2014).

15. Wang, H., Qu, S., Yan, M., Zheng, L. \& Wang, J. Design and analysis of dual-band polarization-selective metasurface. Appl. Phys. A 125, 762 (2019).

16. Cheng, Y., Nie, Y., Wang, X. \& Gong, R. An ultrathin transparent metamaterial polarization transformer based on a twist-split-ring resonator. Appl. Phys. A 111, 209-215 (2013).

17. Huang, C., Feng, Y., Zhao, J., Wang, Z. \& Jiang, T. Asymmetric electromagnetic wave transmission of linear polarization via polarization conversion through chiral metamaterial structures. Phys. Rev. B 85, 195131 (2012).

18. Menzel, C. et al. Asymmetric transmission of linearly polarized light at optical metamaterials. Phys. Rev. Lett. 104, 253902 (2010).

19. Mutlu, M., Akosman, A. E., Serebryannikov, A. E. \& Ozbay, E. Diodelike asymmetric transmission of linearly polarized waves using magnetoelectric coupling and electromagnetic wave tunneling. Phys. Rev. Lett. 108, 213905 (2012).

20. Liu, D., Xiao, Z., Ma, X. \& Wang, Z. Asymmetric transmission of linearly and circularly polarized waves in metamaterial due to symmetry-breaking. Appl. Phys. Express 8, 52001 (2015).

21. Fedotov, V. A. et al. Asymmetric propagation of electromagnetic waves through a planar chiral structure. Phys. Rev. Lett. 97, 167401 (2006).

22. Bokhari, S. H. A. \& Cheema, H. M. Broadband asymmetric transmission via angle-induced chirality enhancement in split ring resonators. J. Appl. Phys. 128, 63102 (2020).

23. Mirzamohammadi, F., Nourinia, J., Ghobadi, C. \& Majidzadeh, M. A bi-layered chiral metamaterial with high-performance broadband asymmetric transmission of linearly polarized wave. AEU Int. J. Electron. Commun. 98, 58-67 (2019).

24. Li, Z., Mutlu, M. \& Ozbay, E. Highly asymmetric transmission of linearly polarized waves realized with a multilayered structure including chiral metamaterials. J. Phys. D. Appl. Phys. 47, 75107 (2014).

25. Khan, M. I. et al. Multiband efficient asymmetric transmission with polarization conversion using chiral metasurface. IEEE Antennas Wirel. Propag. Lett. 19, 1137-1141 (2020).

26. Cheng, Z. \& Cheng, Y. A multi-functional polarization convertor based on chiral metamaterial for terahertz waves. Opt. Commun. 435, 178-182 (2019).

27. Li, S. \& Zhang, X. Asymmetric tri-band linear-to-circular polarization converter in transmission mode. Int. J. RF Microw. Comput. Eng. 30, e21959 (2020).

28. Cheng, Y., Wu, C., Cheng, Z. Z. \& Gong, R. Z. Ultra-compact multi-band chiral metamaterial circular polarizer based on triple twisted split-ring resonator. Prog. Electromagn. Res. 155, 105-113 (2016).

29. Wu, L. et al. Circular polarization converters based on bi-layered asymmetrical split ring metamaterials. Appl. Phys. A 116, 643-648 (2014).

30. Cheng, Y., Nie, Y., Cheng, Z. \& Gong, R. Z. Dual-band circular polarizer and linear polarization transformer based on twisted split-ring structure asymmetric chiral metamaterial. Prog. Electromagn. Res. 145, 263-272 (2014).

31. Zhang, X. \& Li, S. Asymmetric dual-band linear-to-circular converter by bi-layered chiral metamaterial. Int. J. RF Microw. Comput. Eng. 29, e21902 (2019).

32. Khan, S. \& Eibert, T. F. A dual-band metasheet for asymmetric microwave transmission with polarization conversion. IEEE Access 7, 98045-98052 (2019)

33. Yang, X., Fan, D., Ren, A., Zhao, N. \& Alam, M. 5G-based user-centric sensing at C-band. IEEE Trans. Ind. Inform. 15, 3040-3047 (2019).

34. Yang, X. et al. Freezing of gait detection considering leaky wave cable. IEEE Trans. Antennas Propag. 67, 554-561 (2019).

35. Menzel, C., Rockstuhl, C. \& Lederer, F. Advanced Jones calculus for the classification of periodic metamaterials. Phys. Rev. A 82, 53811 (2010).

36. Akbari, M., Farahani, M., Sebak, A. \& Denidni, T. A. Ka-band linear to circular polarization converter based on multilayer slab with broadband performance. IEEE Access 5, 17927-17937 (2017).

37. Mustafa, M.-E., Amin, M., Siddiqui, O. \& Tahir, F. A. Quasi-crystal metasurface for simultaneous half- and quarter-wave plate operation. Sci. Rep. 8, 15743 (2018).

38. Gonçalves, F. J. F., Pinto, A. G. M., Mesquita, R. C., Silva, E. J. \& Brancaccio, A. Free-space materials characterization by reflection and transmission measurements using frequency-by-frequency and multi-frequency algorithms. Electronics 7, 260 (2018).

39. Ahmed, F., Hassan, T. \& Shoaib, N. A multiband bianisotropic FSS with polarization-insensitive and angularly stable properties. IEEE Antennas Wirel. Propag. Lett. 19, 1833-1837 (2020).

\section{Acknowledgements}

This work was supported in part by Engineering and Physical Sciences Research Council grant EP/R511705/1. This work was also partially co-funded by the European Regional Development Fund \& the Republic of Cyprus through the Research and Innovation Foundation, under the project INFRASTRUCTURES/1216/0042 (RFMETA) and Higher Education Commission (HEC) Pakistan-National Research Programme for Universities (NRPU) funded project no. 9971 titled "RF Energy Harvesting for Internet of Things (IoT) Applications".

\section{Author contributions}

S.M.Q.A.S. designed the CPS metasurface, performed the simulations and wrote the initial draft of the manuscript. F.A. and A.Q. contributed to the concept and wrote the relevant text. N.S. performed the measurements and validations. N.S., M.A.I, Q.H.A., A.A. and S.N. supervised the whole research work and reviewed the manuscript.

\section{Competing interests}

The authors declare no competing interests.

\section{Additional information}

Correspondence and requests for materials should be addressed to N.S. or Q.H.A.

Reprints and permissions information is available at www.nature.com/reprints.

Publisher's note Springer Nature remains neutral with regard to jurisdictional claims in published maps and institutional affiliations. 
(c) (i) Open Access This article is licensed under a Creative Commons Attribution 4.0 International cc) License, which permits use, sharing, adaptation, distribution and reproduction in any medium or format, as long as you give appropriate credit to the original author(s) and the source, provide a link to the Creative Commons licence, and indicate if changes were made. The images or other third party material in this article are included in the article's Creative Commons licence, unless indicated otherwise in a credit line to the material. If material is not included in the article's Creative Commons licence and your intended use is not permitted by statutory regulation or exceeds the permitted use, you will need to obtain permission directly from the copyright holder. To view a copy of this licence, visit http://creativecommons.org/licenses/by/4.0/.

(C) The Author(s) 2021 